\title{
Optimal Aircraft Performance During Microburst Encounter
}

\author{
Mark L. Psiaki* \\ Cornell University, Ithaca, New York 14850 \\ and \\ Robert F. Stengelt \\ Princeton University, Princeton, New Jersey 08540
}

\begin{abstract}
The effects of microburst characteristics on the optimal penetration performance of jet transport and general aviation aircraft are presented. The purpose is to determine the best possible performance that can be achieved in a broad range of microbursts. A secondary goal is to illustrate good strategies for dealing with a range of microbursts during takeoff and landing. Over 1100 optimal trajectories were computed for two aircraft types flying through idealized microbursts using a Successive Quadratic Programs trajectory optimization algorithm. Contours of safety metrics are plotted as functions of the length scales, magnitudes, and locations of horizontal wind shears and vertical downdrafts. These performance contours show three length-scale regimes for optimal microburst penetration. At short length scales, hazards usually associated with gustiness predominate (e.g., high normal load factor, rotational upset). At intermediate length scales, a degraded ability to maintain flight path and/or vertical velocity poses the most serious threat. At very long microburst length scales, excessive touchdown velocities may result. The ability to transit a microburst successfully also varies strongly with microburst location. The results show that both aircraft types could penetrate some very severe microbursts if optimal control histories were followed. Nevertheless, these control strategies assume perfect prior knowledge of the wind, and practical limits to successful encounter with real-time control capabilities would be lower. The optimally controlled jet transport can successfully penetrate higher intensity microbursts than can the general aviation aircraft.
\end{abstract}

\section{Introduction}

O N August 2, 1985, a Delta Airlines Lockheed L-1011 crashed while attempting to land at the Dallas/Ft. Worth Airport because it encountered a severe microburst wind shear (like that in Fig. 1). The flight data records ${ }^{\mathrm{l}}$ provide insight into the combination of wind conditions and control strategy that caused this accident. During the headwind portion of the microburst, the aircraft was pitched up, apparently in an attempt to reduce airspeed. Because of the accompanying downdraft, it failed to experience much altitude gain, but it did experience a significant groundspeed loss. After regaining the initial approach airspeed, the aircraft kept losing airspeed rapidly because it entered the tailwind portion of the microburst. When the airspeed got within $10-15 \mathrm{kt}$ of the stall speed, it was pitched down, apparently in an attempt to regain airspeed. It did regain airspeed, but in the process, it crashed into a highway $1.1 \mathrm{~nm}$ short of the runway. This is a classic example of how the conventional piloting strategy of maintaining airspeed with the elevator can spell disaster in a microburst encounter.

With this experience (and others like it) in mind, a number of efforts have attacked the microburst encounter problem from the perspective of improving control strategy. ${ }^{2-16}$ Each control strategy realized significant performance improvements over existing systems in test cases. They did this by using pitch (and, in some cases, thrust) inputs to counteract the microburst's disturbing effects on such quantities as altitude and airspeed. Methodologies ranging from classical feed-

Presented as Paper 88-4367 at the AIAA 15th Atmospheric Flight Mechanics Conference, Minneapolis, MN, Aug. 15-17, 1988; received Jan. 9, 1989; revision received Feb. S, 1990 . Copyright (C) 1990 by the American Institute of Aeronautics and Astronautics, Inc. All rights reserved.

*Assistant Professor, Mechanical and Aerospace Engineering. Member AIAA.

†Professor, Mechanical and Aerospace Engineering. Associate Fellow AIAA. back design ${ }^{3,11}$ to deterministic trajectory optimization ${ }^{12.15,16}$ have been used. In fact, many of the control schemes were able to safely penetrate wind profiles that had caused serious accidents. This improved performance is encouraging information, but in most cases, the actual number of simulated wind shear encounters was small for each control law, so more extensive testing is clearly in order. Effects of variations in microburst intensity were considered in Refs. $9,12,15$, and 16 , whereas the effects of differing aircraft performance characteristics were treated in Refs. 12 and 16.

The present work is part of an extended effort to address the problem or to improve piloting strategies for microburst encounter. , $11,12.17 .18$ The effort began with a classic control design study ${ }^{11}$ and progressed to deterministic optimization of encounters with Doppler-radar-derived microburst wind fields. ${ }^{12}$ The present paper characterizes the dependence of deterministically optimized penetration trajectories on microburst properties, including length scale, intensity, location, and relative magnitude of horizontal vs vertical winds. This dependence shows how different microbursts pose different types and levels of threat to an optimally controlled aircraft. The basic method of determining this dependence is to compute many deterministic optimal trajectories through a parametric family of microbursts. Results are presented for two different aircraft types, a small general aviation aircraft (GA)

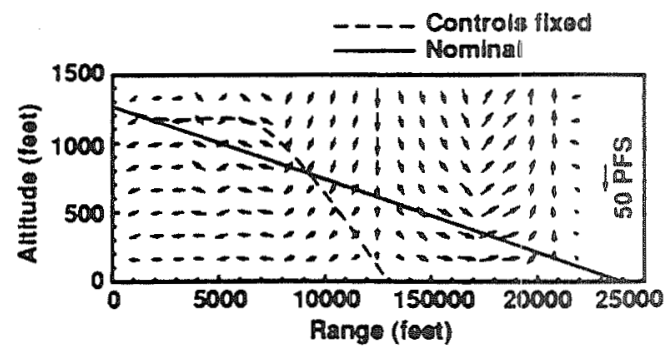

Fig. 1 Two JT approach trajectories through the most severe Joint Airport Weather Studies headwind tailwind shear. 
and a $150,000-1 \mathrm{~b}$ jet transport (JT). The takeoff and landingapproach flight phases are both considered. Reference 17 contains further results in this area.

As a side benefit, the numerous trajectory optimizations of this study point to possible strategy improvements that could be realized by practical control systems, as noted. Reference 18 follows up on this idea, presenting simplified, realizable implementations of these strategies. It is similar to efforts of Miele er al., who have also used optimization results to deduce practical strategies. ${ }^{7.8 .13}$

\section{Analysis Highlights}

\section{Microbursi Encounter Modelin}

The longitudinal motions of an aircraft were modeled by seven coupled, scalar, nonlinear, ordinary differential equations that solve for inertial speed $V_{i}$, flight-path angle $\gamma_{i}$, pitch rate $q_{i}$, angle of attack $\alpha_{i}$, altitude $h$, range $r$, and thrust or power $T$ or $P$ where $\hat{\delta} E$ is the elevator setting, $\hat{q}=q \bar{c} / 2 V_{a}, \bar{\alpha}=\alpha \bar{c} / 2 V_{1}$, and $\bar{c}$ is the mean aerodynamic chord. Table $l$ gives the coefficient values used in this study. Much of the JT aeordynamic model was taken from Ref. 19. The GA coefficients are based on data in Ref. 20. Note that the available $C_{L_{\text {max }}}$ of each model can be derived from the $C_{L_{0}}, C_{L_{\alpha}}$, and $\alpha_{\max }$ data in Table I; $C_{L_{\max }}=2.50$ for the JT; $C_{L_{\max }}=1.77$ for the GA. Trajectory optimization inequality constraints enforce these $C_{L_{\max }}$ values by enforcing the inequality constraint $\alpha_{a} \leq \alpha_{\max }\left(<\alpha_{\text {stail }}\right)$. Enforcing an $\alpha_{\max }$ slightly less than $\alpha_{\text {stall }}$ (ulimate maximum of $C_{L}$ ) decreases the likelihood of departure from controlled flight. Note also that the zero lift line occurs at negative angle of attack in both cases, representing flaps-down configurations. Zero lift of the JT configuration occurs at $\alpha=-15.5$ deg because of the high value of $C_{L_{0}}$ taken from Ref. 19; hence, JT negative angle-of-attack time histories appear anomalous (as in Fig. 4), but they correspond to positive $C_{L}$.

$$
\begin{aligned}
\dot{V}_{i}= & \frac{\left[-\bar{q} S\left[C_{D} \cos \left(\alpha_{i}-\alpha_{a}\right)+C_{L} \sin \left(\alpha_{i}-\alpha_{a}\right)\right]+T \cos \left(\alpha_{i}+i_{T}\right)\right]}{m}-g \sin \gamma_{i} \\
\dot{\gamma}_{i} & =\frac{\left[\frac{\bar{q} S\left[C_{L} \cos \left(\alpha_{i}-\alpha_{a}\right)-C_{D} \sin \left(\alpha_{i}-\alpha_{a}\right)\right]+T \sin \left(\alpha_{i}+i_{T}\right)}{m}\right]-g \cos \gamma_{i}}{V_{i}}
\end{aligned}
$$$$
\dot{q}_{i}=\frac{\bar{q} S \bar{c} C_{M}}{I_{y y}}
$$$$
\dot{\alpha}_{i}=q_{i}-\dot{\gamma}_{i}
$$$$
\hat{h}=V_{i} \sin \gamma_{i}
$$$$
\dot{r}=V_{i} \cos \gamma_{i}
$$

and

$$
\begin{array}{r}
\dot{T}=\frac{(\delta T-T)}{\tau_{e}} \quad \text { (JT aircraft) } \\
\dot{P}=\frac{(\delta T-P)}{\tau_{e}} \quad \text { with } T=\frac{E\left(V_{a}\right) P}{V_{a}}
\end{array}
$$

Here, the reference area is $S, g$ represents gravitational acceleration, $m$ and $I_{y y}$ are aircraft mass and pitching moment of ineria, $i_{T}$ is the thrust incidence angle, and $\delta T$ is the throttle setting. The time constant for thrust (or power) ' $-3 \tau_{e}$ is $4 \mathrm{~s}$ for the jet transport and $1 \mathrm{~s}$ for the general aviation aircraft; $E\left(V_{a}\right)$ represents the airspeed-dependent propeller efficiency of the general aviation aircraft. The wind shear effects on the aircraft's motions enter through the air-relative angle of attack $\alpha_{a}$ and the airspeed $V_{a}$,

$$
\begin{gathered}
\alpha_{a}=\alpha_{i}+\gamma_{i}-\tan ^{-1}\left(\frac{V_{i} \sin \gamma_{i}+w_{v}}{V_{i} \cos \gamma_{i}+w_{h}}\right) \\
V_{a}=\sqrt{V_{i}^{2}+w_{v}^{2}+w_{h}^{2}+2 V_{i}\left(w_{v} \sin \gamma_{i}+w_{h} \cos \gamma_{i}\right)}
\end{gathered}
$$

and the dynamic pressure is computed as $\bar{q}=\rho V_{a}^{2} / 2$.

The aerodynamic models for pitching moment, lift, and drag coefficients $C_{M}, C_{L}$, and $C_{D}$ are quasisteady models that are reasonably accurate at low Mach number and below the stall angle of attack

$$
\begin{gathered}
C_{M}=C_{M_{0}}+C_{M_{\alpha}} \alpha_{a}+C_{M_{B E}} \delta E+C_{M_{q}} \hat{q}_{i}+C_{M_{\dot{\alpha}}} \hat{\dot{\alpha}}_{a} \\
C_{L}=C_{L_{0}}+C_{L_{\alpha}} \alpha_{a}+C_{L_{3 E}} \delta E+C_{L_{q}} \hat{q}_{i}+C_{L_{\dot{\alpha}}} \hat{\dot{\alpha}}_{a} \\
C_{D}=C_{D_{0}}+\epsilon C_{L}^{2}
\end{gathered}
$$

The $C_{L_{\max }}$ is the significant quantity that limits an aircraft's ability to safely negotiate a microburst wind shear, and it takes on reasonable values for both configurations.

The seven scalar differential equations can be put in the form $\dot{x}=f(x, u, w)$ by defining the state vector, $x=\left(V_{i}, \gamma_{i}, q_{i}\right.$, $\alpha_{i}, h, r, T$ or $\left.P\right)^{T}$, the control vector, $u=(\delta E, \delta T)^{T}$, and the disturbance vector, $w=\left(-w_{h},-w_{v}\right)^{T}$. Except for the $w$ dependence, this form is a special case of the form needed for trajectory optimization.

For the present study, the microburst wind field was modeled as a function of range along the intended flight corridor; our prior optimization study considered altitude-dependent profiles measured in the Joint Airport Weather Studies (JAWS) program as well. ${ }^{12}$ Five parameters characterize the engineering approximation model: the wave length and intensity of a sinusoidal headwind/tailwind, the wave length and intensity of a (1-cosine) downdraft, and the location of the microburst core (Fig. 2). This model has been found to produce aircraft trajectories like those through the JAWS microburst profiles. ${ }^{12,17} \mathrm{Headwind} /$ tailwind and downdraft effects were considered separately so that parametric trends could be identified.

\section{Trajectory Optimization}

The trajectory optimization problem is of the continuoustime, fixed-end time, free-end-point, Bolza type.

Find

$$
u(t) \text { for } t \in\left[t_{0}, t_{f}\right]
$$

Table 1 Aerodymamic properties of the Jet Transport (JT) and Gemeral Aviation (GA) Models

\begin{tabular}{ccccccccc}
\hline \hline Aircraft & $C_{L_{0}}$ & $C_{L_{\alpha}}$ & $C_{L_{\delta E}}$ & $C_{L_{\phi}}$ & $C_{L_{\dot{\alpha}}}$ & $\begin{array}{c}\alpha_{\max } \\
\text { deg }\end{array}$ \\
\hline $\mathrm{JT}$ & 1.36 & 5.04 & 0.40 & 9.3 & 6.6 & 13 \\
$\mathrm{GA}$ & 0.61 & 4.73 & 0.48 & 15.9 & 8.0 & 14 \\
& $\cdot$ & & & & & & \\
Aircraft & $C_{M_{0}}$ & $C_{M_{\alpha}}$ & $C_{M_{\delta E}}$ & $C_{M_{Q}}$ & $C_{M_{\dot{\alpha}}}$ & $C_{D_{0}}$ & $\epsilon$ \\
\hline $\mathrm{JT}$ & 0.0 & -1.21 & -1.43 & -29.5 & -17.7 & 0.064 & 0.067 \\
$\mathrm{GA}$ & -0.09 & -0.93 & -1.35 & -24.6 & -12.3 & 0.066 & 0.055 \\
\hline \hline
\end{tabular}




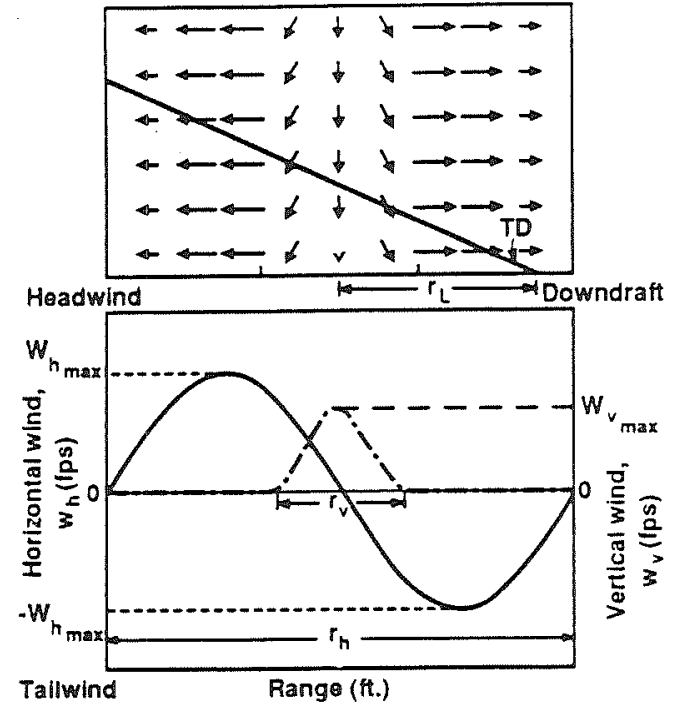

Fig. 2 An engineering-approximation wind shear model: a) vertical cross-section; b) variation of horizontal and vertical winds. to minimize

$$
J=\int_{t_{0}}^{t_{f}} L[x(t), u(t), t] \mathrm{d} t+V\left[x\left(t_{f}\right), t_{f}\right]
$$

subject to

$$
\begin{gathered}
\dot{x}=f(x, u, t) \\
x\left(t_{0}\right)=x_{0} \\
c[x(t), u(t), t] \leq 0 \text { for all } t \in\left[t_{0}, t_{f}\right]
\end{gathered}
$$

where the typical $L=\left\{2\left[\left(h-h_{0}\right) \cos \gamma_{i 0}-\left(r-r_{0}\right) \sin \gamma_{i 0}\right]^{2}\right.$ $\left.\left.+0.04\left[\delta E-\delta E_{0}\right]^{2}+0.004\left[\delta T-\delta T_{0}\right]^{2}\right)\right\}$ and $V=1 / 2 \Delta x_{f}^{T} P_{N} \Delta x_{f}$; they penalize deviations from the intended flight path. In these cost function expressions distances are expressed in feet, elevator angles in degrees, and throttle settings in percent of maximum. The quantities $h_{0}$ and $r_{0}$ are initial values; $\gamma_{i 0}, \delta E_{0}$, and $\delta T_{0}$ are nominal values. The vector $\Delta x_{f}$ is the final state perturbation from the nominal flight condition and flight path, and the matrix $P_{N}$ is the solution of a discrete-time algebraic Riccati equation involving the sampled-data linearization of Eq. (1) about the nominal flight condition and involving the cost function integrand $L$. The function $f$ describes the nonlinear longitudinal motions of an aircraft in a wind shear, $c$ describes state and control inequalities that must be enforced for the optimal solution to make physical and practical sense (e.g., throttle saturation and stall angle of attack), $x_{0}$ gives the initial position and flight condition, and $t_{0}$ and $t_{f}$ ensure that the trajectory traverses the entire microburst.

The function $f$ contains global information about the wind shear because this is a deterministic formulation of the trajectory optimization problem; therefore, the optimal solution "knows" ahead of time what the microburst is going to do. This foreknowledge of the wind field makes the selection of $x_{0}$ important. If the initial range location is farther ahead of the center of the microburst, the optimization will have more time to prepare for the encounter. For example, the optimization might call for the airplane to put the throttle forward to store excess airspeed in preparation for coming tailwinds even before the airplane actually enters the tailwind section.

In this study, the initial range location is just at the onset of any winds, at the beginning of the headwind build-up section if $w_{h_{\max }} \neq 0$, at the beginning of the downdraft build-up section otherwise. The initial airspeed is the nominal airspeed during takeoff and landing. By comparison. Miele er al. $210.1 \%$ and Zhao and Bryson's assume that the initial range location is at the maximum headwind and that the initial airspeed is at the nominal. In their optimal trajectories, the aircraft has less time to prepare for the microburst tailwinds, and it starts with a deficit in inertial speed. Consequently, for given peak-topeak winds, their results are more conservarive.

A second difference is in the cost functions optimized here and in the referenced studies. Whereas we have minimized a quadratic path-following error, Miele et al..$^{9,10,16}$ and $Z$ hao and Bryson ${ }^{15}$ minimized the maximum altitude or altitude rate deviations that occurred on the trajectories. The minimummaximum and quadratic altitude deviation cost functions yield similar optimal trajectories. Zhao and Bryson also investigated a cost function based on final air-relative total energy, which produces very different optimal trajectories.

Solutions to the trajectory optimization problem in Eq. (4) have been obtained by application of the method of Successive Quadratic Programs (SQP) to a discrete-time approximation of the original problem. ${ }^{17}$ The discretization is like that of a zero-order hold: it breaks the interval from $t_{0}$ to $t_{f}$ into $N$ equal subintervals, $t_{k}$ to $t_{k+1}$ for $k=0 \ldots N-1 ; t_{N}=t_{f}$. The controls are held fixed over each subinterval, and the continuoustime inequality constraint is enforced only at the sampling times $t_{k}$. The optimization used 70 sampling intervals per microburst encounter.

Parametric Study of Effects of Microburst Variations

Optimal trajectories were computed for various values of the microburst parameters, $r_{h}, w_{h_{\max }}, r_{v}, w_{v_{\max }}$, and $r_{L}$, for two aircraft types, and for takeoff and approach flight paths. Each parameter combination leads to a different optimal microburst encounter trajectory. Several quantities that characterize the safety of the encounter were extracted from each trajectory and were associated with a particular point in microburst parameter space. Thus, altitude deviation, sink rate, minimum airspeed, and other measures of the trajectories can be portrayed as functions of the microburst parameters, and acceptable limiting values can be expressed in terms of these parameters. The resulting plots present standards against which practical sub-optimal controllers can be judged.

Altitude deviation on landing approach leads to touchdown-point deviation. Vertical velocity deviations can be hazardous on both climb-out and approach. On approach, too high a sink rate at touchdown can cause the aircraft structure to fail. If the ascent rate is too small or negative on climb-out, eventual obstacle impact becomes likely. Airspeed-deviation hazards, as considered here, are those associated with the loss of rotational stability and control through the loss of aerody. namic moments; it could become the critical safety factor in very intense, very short length-scale microbursts. The $1-g$ stall limit on airspeed is not considered as a safety factor because stall has been directly factored into the trajectory optimizations as a state inequality constraint on angle of attack. Inertial velocity deviations during landing approach are considered because of the necessity to stop the airplane in the given runway length after landing. ${ }^{21}$

This study examines headwind/tailwind $(H / T)$ effects and downdraft (DD) effects separately. The separation reduces the scope of our parametric study to a feasible level. An actual microburst has both horizontal and vertical wind components. For a predominantly $\mathrm{H} / \mathrm{T}$ microburst encounter (as happens very near the ground), our study of pure $H / T$ encounter gives a good prediction of the trends. Also, for a predominantly $D D$ microburst encounter (as happens further aloft), our study of pure DD encounter gives a good prediction of the trends. For a mixed H/T-DD microburst encounter, superposition of actual trajectory response applies up to a certain level of wind activity, but superposition makes no sense in reference to maximal performance contours. Nevertheless, the performance study of the separate cases gives insight into the quali- 


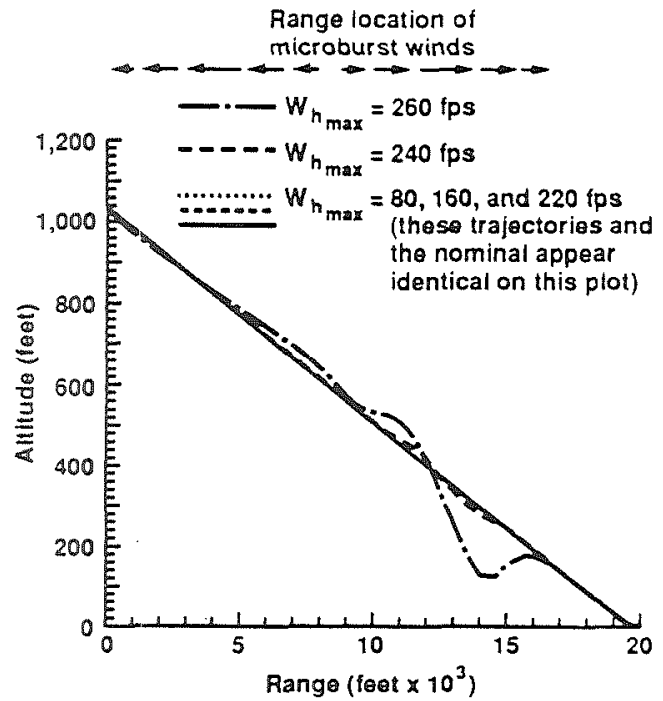

Fig. 3 Optimal JTr landing-approach trajectories through five headwind tailwind microbursts of varying intensity $\left(r_{h}=16,000 \mathrm{ft}\right)$.

tative trends in strategy and performance that can be expected in the mixed case.

The dependence on microburst location, $r_{L}$, was suppressed for the H/T and DD results. Instead of fixing the value of $r_{L}$, $r_{L}$ takes its worst-case value with respect to any given safetyrelated quantity. Suppose $q_{i}$ is the quantity in question and that higher values of the quantity indicate a deterioration of safety, then the value of the quantity used in the $H / T$ cross section would be

$$
q_{i_{\max }}\left(r_{h}, w_{h_{\max }}\right)=\max _{r_{L}} q_{i}\left(r_{h}, w_{h_{\max }}, r_{v}=0, w_{v_{\max }}=0, r_{L}\right)
$$

Variations of $r_{L}$ do not affect the optimal control actions in the analysis of this paper because flare and pre-takeoff runway roll have not been considered. Microburst location variations only affect the location of the penetration trajectory with respect to the ground. Therefore, maximizations as in Eq. (5) can be carried out over a single optimal trajectory, i.e., by computing a single trajectory for a given $r_{h}$ and $w_{h_{\max }}$ and maximizing $q_{i}$ over that trajectory. Effects of microburst core location, $r_{L}$, also were considered separately.

\section{Trajectory Optimization Results}

Over a thousand optimal microburst encounters have been computed. The optimal trajectories fall broadly into eight distinct categories, each distinct combination of aircraft type (IT or GA), wind feature (DD or $H / T$ ), and flight phase (landing approach or takeoff). In each of these eight categories, 60-210 distinct optimal trajectories were computed at grid points of microburst intensity vs length scale. The microburst length scales range from shorter than the distance traveled during one short period to longer than the distance traveled during one phugoid period. The microburst wind intensities range up to hundreds of $\mathrm{ft} / \mathrm{s}$; details for all of these trajectories, can be found in Ref. 17.

The solution of a trajectory optimization problem is a statespace trajectory and its associated control time history. Figures 3 and 4 present examples of aircraft-in-a-microburst optimal trajectories. The JT aircraft is used in these examples, the microbursts are $\mathrm{H} / \mathrm{T}$ microbursts, the primary cost function component is mean-square altitude deviation, and the flight phase is landing approach. On the altitude vs range plots (Fig. 3), three of the optimal trajectories are indistinguishable from the nominal trajectory. The plots of associated time histories (Fig. 4) show significant deviations from nominal in all cases. These solutions provide good examples of the information contained in an optimal trajectory.
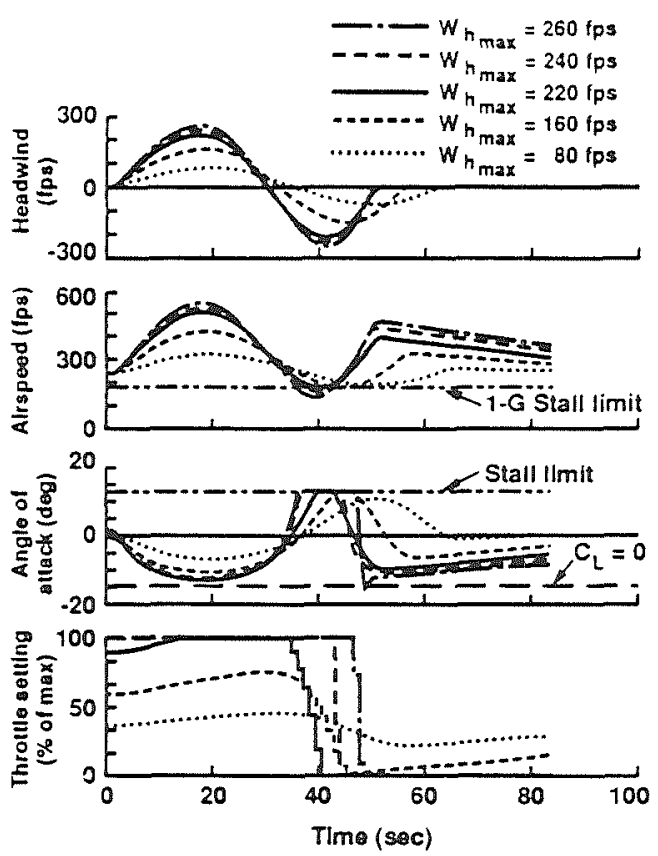

Fig. 4 Time histories associated with five optimal JT landing approaches through headwind/tailwind microbursts of varying intensity $\left(r_{h}=16,000 \mathrm{ft}\right)$.

Insight into the characteristics of good control in a microburst has been a side benefit of this parametric study. In Figs. 3 and 4 , unconventional pitch steering maintains lift, thereby maintaining glide path-note the 180 deg phase shift between the airspeed and angle-of-attack time histories on Fig. 4. As the microburst intensity increases, this strategy eventually leads to saturation of the angle of attack at the stall limit. The negative angles of attack indicated on Fig. 4 still correspond to positive lift; note the line indicating the zero-lift angle of attack. Throttle activity increases to avoid airspeed excursions below the $1-g$ stall limit, and microburst transit occurs more quickly because of the resultant higher inertial velocities. These combined strategies do a good job of maintaining the intended glide path up to a microburst intensity of $220 \mathrm{ft} / \mathrm{s}$ (corresponding to a peak-to-peak variation of 440 fps).

The throttle setting increases from the nominal value in the headwind zone in all cases of Figs. 3 and 4 as a consequence of the global knowledge assumed during optimization. In other words, the optimization algorithm benefits from predictive information that would require "look ahead" measurements in the aircraft or "uplink" of ground-based measurements of the wind field. Reference 18 presents a related real-time throtthe strategy that does not require predictive capability.

The control strategies used by all of the optimizations of this study have the following common characteristics. They use pitch control in an unconventional manner to maintain lift while using throttle to keep from running out of airspeedtailwinds steal airspeed directly and downdrafts steal energy, which is equivalent to airspeed. When an extreme microburst causes the airspeed to fall below the $1-g$ stall limit, despite counteracting throttle activity, the unconventional strategy is continued: the angle of attack is held at the stall limit until the microburst subsides. The net altitude loss due to this maneuver is less than what would be caused by diving to regain airspeed. The current results suggest that $\alpha$ be raised to the stick-shaker limit, where $C_{L}$ is very high but not yet at its ultimate maximum and where the likelihood of lateral-directional control problems still is quite low. By bleeding off excess airspeed in favor of altitude down to the stick-shaker limit, an aircraft may ride out a severe microburst transient without deviating appreciably from its intended flight path. 
Performance as a Function of Microburst Parameters

Landing Approach Performance Through Headwind/ Tailwind Microbursts

Figures 5 and 6 present limiting $\mathrm{H} / \mathrm{T}$ intensities as functions of length scale for the JT and GA aircraft executing landing approach. The figures also present an approximate curve for the altitude deviation performance. The symbols on the plots refer to points of the contours that have been computed via interpolation with respect to microburst intensity. Extrapolations beyond the symbols indicate that some data exist at the adjacent length scale, but not at high enough microburst intensity to determine the next interpolation point. The microburst length scales at which optimal trajectories have been computed range from 250 to $16,000 \mathrm{ft}$ for the GA aircraft and from 1000 to $64,000 \mathrm{ft}$ for the JT aircraft on a logarithmic grid centered near the respective aircraft's phugoid length scale. The different intensity scales of the two plots reflect the lower ability of the GA aircraft to handle severe microburst winds.

The performance contours for the two aircraft display several similarities. The primary safe-performance measure, the maximum altitude deviation, has contours that are concave upward on both plots for higher values of the maximum deviation. The minimum intensity for a given contour occurs at a microburst length scale between that of the short period mode (GA: $230 \mathrm{ft}, \mathrm{JT}: 1800 \mathrm{ft}$ ) and that of the phugoid mode (GA: $2510 \mathrm{ft}, \mathrm{JT}: 10,470 \mathrm{ft}$ ). The maximum-altitude-deviation contours are close together at long microburst length scales but diverge at short microburst length scales.

At short microburst length scales, the minimum-airspeed limit becomes important. The minimum-airspeed contours reach high microburst intensities for the longer length-scale microbursts. At short microburst length scales, however, the microburst intensities of the minimum-airspeed contours decrease while the intensities of other contours increase, thus making the minimum airspeed limit a critical factor in this regime. As a point of reference, the $1-g$ stall airspeeds are 180 and $85 \mathrm{ft} / \mathrm{s}$ for the JT and GA aircraft, respectively.

At longer microburst length scales, the upper limit on inertial velocity may become a crucial limit. These contours do just the reverse of the minimum-airspeed contours: they are at high microburst intensity for short microburst length scale,

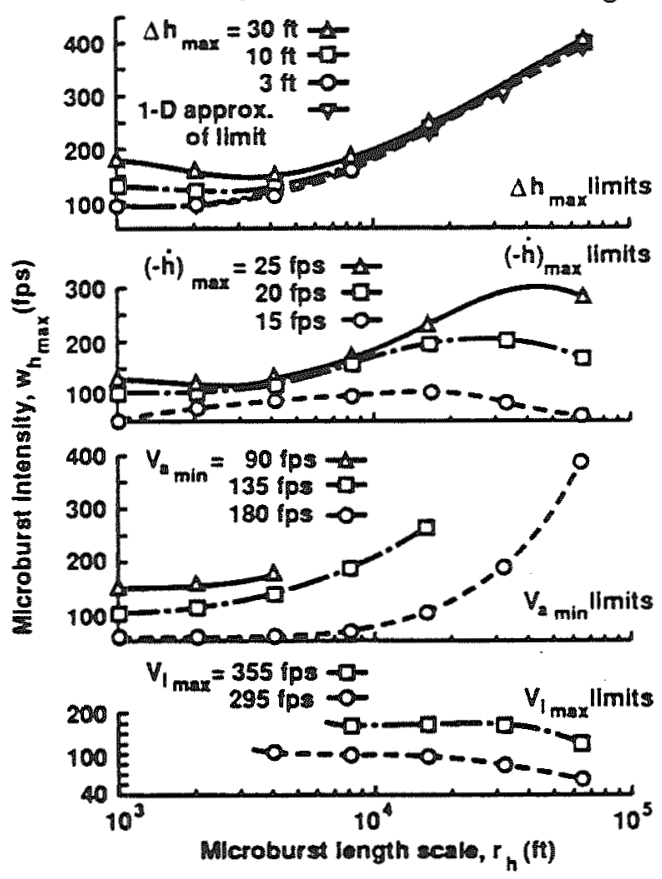

Fig. 5 Performance safety contours for optimal JT landing approach in headwind/taillwind microbursts. but their intensities decrease with increasing microburst length scale. Recalling that a limit to inertial velocity arises from braking-distance/runway limitations, one can compute reasonable values for this limit based on flight manuals such as in Ref. 21.

In contrast to the other contours, the maximum-descentrate contours for the two aircraft (the second graph from the top on each of Figs. 5 and 6) are dissimilar. As a point of reference, the nominal descent rates are 12 and $6 \mathrm{ft} / \mathrm{s}$ for the JT and GA aircraft. Contours corresponding to a maximum descent rate that is double the nominal descent rate fall in regions of microburst parameter space where other safety factors would allow a landing approach, e.g., $w_{h_{\max }}=120 \mathrm{fps}$, $r_{h}=3000 \mathrm{ft}$ for JT penetration, and $w_{h_{\max }}=60 \mathrm{fps}, r_{h}=1000$ $\mathrm{ft}$ for the GA penetration. Descent-rate limits must be considered to determine the safety of landing approach in a $\mathrm{mi}$ croburst.

Some of the trends of these performance safety contours have simple explanations. A dynamic, one-dimensional analysis agrees well with the long length-scale altitude-deviation performance contours. References 17 and 18 explain this analysis, which is a dynamic version of the analysis used to generate an aircraft's power curve from its lift, drag, and thrust characteristics. Stall also plays a role in this analysis, which estimates microburst-induced airspeed variations that occur while tracking the glide path. The airspeed variations are estimated by integrating a differential equation for $\mathrm{d} V / \mathrm{d} r$ that is derived under the assumptions of $100 \%$ throttle setting and perfect glide-path-maintaining pitch control. On the maximum-altitude-deviation contour graphs (top graphs) of Figs. 5 and 6, the curves marked "1-D Approx. of Limit" were generated from this analysis. For long length-scale $H / T$ microbursts, these curves agree well with the actual maximum-altitude-deviation contours.

The maximum inertial-velocity contours can be explained in terms of the general strategy that has been described above. The trajectory optimizations try to avoid excursions of the airspeed below the $1 \mathrm{~g} g$ stall limit by accelerating the aircraft

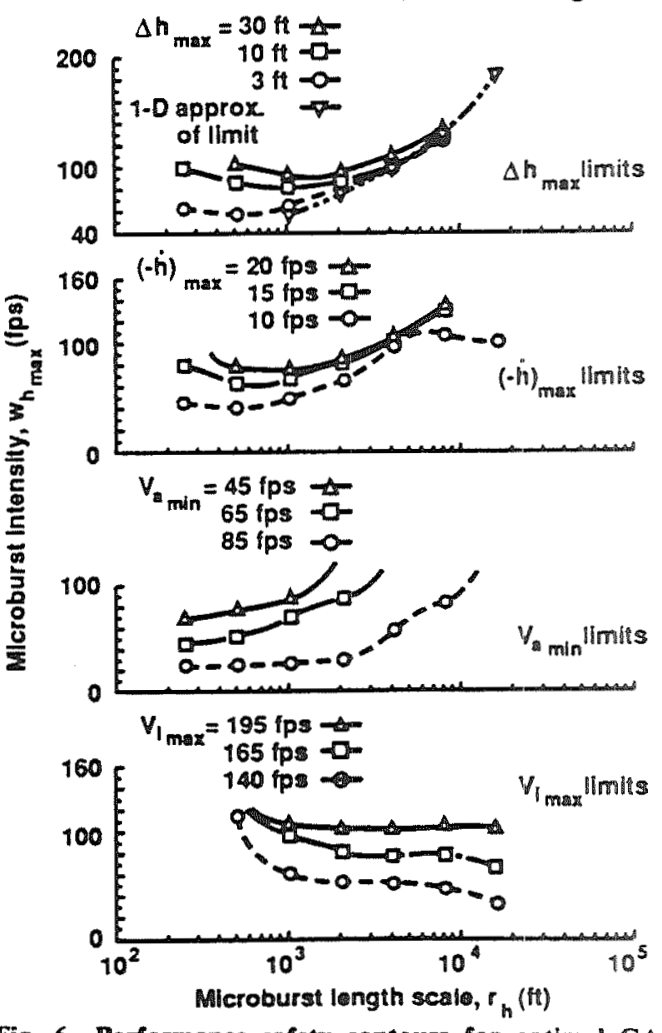

Fig. 6 Performance safety contours for optimal GA landing approach in headwind/tailwind microbursts. 


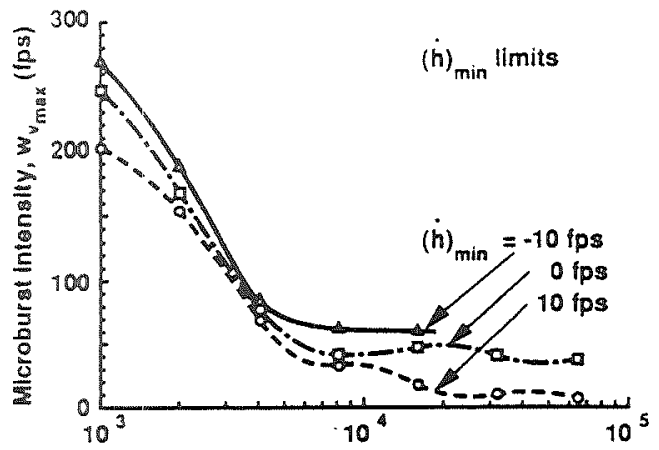

Fig. 7 Performance safety contours for optimal $\mathbf{J T}$ climb-out in downdraft microbursts.

with thrust. This strategy becomes more and more effective for longer length-scale microbursts because the thrust has more time to work. Increased inertial velocities in the longer length-scale microbursts go hand in hand with this increased ability to maintain flight path, thus the downward trend of the inertial-velocity contours at long microburst length scales: higher inertial-velocity maxima occur in less severe microbursts. At very long length scales, the throttle is fully capable of maintaining airspeed, and penetration safety is simply a function of the ability of the aircraft to land in a steady tailwind. At microburst length scales shorter than the phugoid length scale, on the other hand, the aircraft cannot change its inertial velocity much due to its large mass; therefore, these contours go to very high microburst intensities and become irrelevant to the question of safety.

For microburst length scales below the phugoid length scale, an aircraft's inertial-velocity cannot vary significantly, and the maximum airspeed loss equals the maximum tailwind. At these short length scales, the microburst intensity of each minimum-airspeed contour is approximately the difference between the nominal airspeed and the contour airspeed.

In summary, the performance safety contours for optimal aircraft landing approach in $H / T$ microbursts display three regimes. In the shortest length-scale regime, shorter than the scale of the aircraft short-period mode, the hazard of upset associated with very low airspeed and loss of aerodynamic control torque is the most restrictive safety criterion. The ability to maintain aircraft altitude is one of the two most important factors in the length-scale regime between the shortperiod mode and the phugoid mode. The ability to maintain slow descent rate is the other important factor in this regime. The maximum allowable inertial velocity at touchdown can limit the ability to successfully negotiate $\mathrm{H} / \mathrm{T}$ microbursts in the length-scale regime above the phugoid mode.

Takeoff Performance of a Jet Transport in Downdraft Microbursts

Figure 7 presents results for JT climb-out through downdraft microbursts. The meanings of symbols and the contour plotting methods have been carried over from the previous two figures. For comparison's sake, the same flaps-down aircraft configuration has been used for this study as for the landing approach study, even though this is not the standard takeoff configuration.

In this case, the minimum ascent rate is the critical performance safety factor over the entire length-scale regime that has been considered. At microburst length scales longer than the phugoid length scale, most of the minimum-ascent-rate contours level out and approach a microburst intensity equal to the maximum static ascent rate less the minimum ascent rate associated with the particular contour. At length scales below the phugoid length scale, the microburst intensities of the contours increase with decreasing length scale.

Reference 17 presents the other five sets of intensity/lengthscale performance safery contours for optimal microburst encounter. They are similar in structure to the three sets pre-

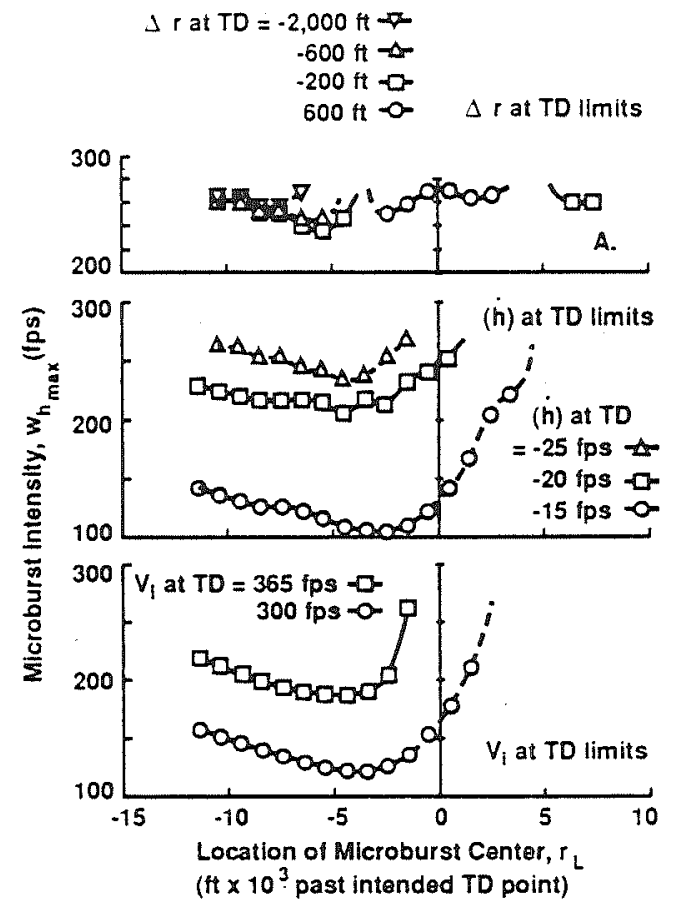

Fig. 8 Performance safety contours for optimal JT landing approach as functions of microburst core location and headwind/tailwind intensity $\left(r_{h}=16,000 \mathrm{ff}, w_{v_{\max }}=0\right)$.

sented here, but shed additional light on the questions of what factors limit safety in what regimes and why they limit it.

Location-Dependent Safety Contours: $A$ Landing-Approach Example

The gross effects of microburst location were evaluated by shifting the core of $\mathrm{H} / \mathrm{T}$ profiles and examining changes in touchdown parameters, neglecting flare dynamics. Actual touchdown values would be affected by flare but would follow the trends shown here.

Figure 8 presents three safety-measure contours for the case of optimal JT landing approach through $\mathrm{H} / \mathrm{T}$ microbursts of length scale $r_{h}=16,000 \mathrm{ft}$. The horizontal axes of the graphs give microburst location relative to the intended touchdown point, $r_{L}$. Positive location indicates that the microburst center occurs beyond the intended touchdown point (i.e., the aircraft is nominally on the runway). The vertical axes give microburst intensity, $w_{h_{\max }}\left(w_{v_{\max }}=0\right)$. The top graph on the figure presents contours of constant range deviation at touchdown. The $+600 \mathrm{ft}$ contour indicates cases that overshoot the intended touchdown point by $600 \mathrm{ft}$ (land 600 -ft long); the negative-valued contours correspond to cases that produce short landings.

The range-deviation-at-touchdown contours indicate that the worst-case microburst location for producing short landings has the center of the outflow occurring 5000 to $9000 \mathrm{ft}$ before the aircraft reaches its intended touchdown point. Given the microburst length scale of $16,000 \mathrm{ft}$ and its sinusoidal $\mathrm{H} / \mathrm{T}$ variation, the maximum tailwinds occur 1000 to $5000 \mathrm{ft}$ before the intended touchdown point. This makes sense; the tailwinds produce the hazard in $H / T$ microbursts, and if they are to cause an aircraft to land significantly short, they must occur far enough before the runway threshold without being so far from the runway as to allow for recovery before ground impact occurs.

The descent-rate contours and the inertial-velocity contours also have the worst-case location for the microburst center occurring before the aircraft reaches its intended touchdown point. These worst-case locations are 2000 to $5000 \mathrm{ft}$ before the intended touchdown point for the descent rate-at-touchdown criterion and 3000 to $5000 \mathrm{ft}$ before it for the inertial-velocity-at-touchdown criterion. These criteria have the worst- 
case microburst occurring closer to the airport than does the range-deviation-at-touchdown criterion.

\section{Conclusions}

This paper has presented and discussed the results of a study of the dependence of optimal aircraft penetration trajectories on microburst wind shear characteristics. The method of study plots contours of safety metrics in a parameter space of microburst characteristics. Optimal performance has been studied for two aircraft, a jet transport and a general aviation aircraft, and for two flight phases, climb-out and landing approach.

For optimally controlled aircraft, performance safety on landing approach is limited in medium length-scale microbursts primarily by the aircraft's ability to track the nominal glide path or to maintain low descent rate at touchdown. In long-length-scale microbursts, a critical limit to safe performance on landing approach is the aircraft's maximum touchdown inertial velocity. Safe performance on climb-out is limited primarilty by the aircraft's ability to continue climbing in the microburst. For short length-scale microbursts with a large tailwind, a factor that may limit safe performance is the danger of losing aerodynamic control torque at very low airspeeds. Microburst location also has a strong effect on the safety of an encounter. On landing approach, the most threatening peak tailwind locations for an optimally controlled aircraft occur from zero to one mile before the runway threshold.

The rerorted results have been achieved by optimal strategies that tightly control altitude via elevator inputs and that use throttle inputs to keep airspeed above the $1 \mathrm{~g}$ stall limit. These strategies track the intended flight path by taking advantage of transient phenomena and by trading airspeed for altitude down to the stick-shaker limit.

\section{Acknowledgments}

This research was supported in part by the Federal Aviation Administration and NASA under Grant NGL 31-001-252.

\section{References}

'Bach, R. E., Jr., and Wingrove, R. C., "The Analysis of Airline Flight Records for Winds and Performance with Application to the Delta 191 Accident," AIAA Paper 86-2227, 1986.

${ }^{2}$ Rynaski, E. G., and Govindaraj, K. W., "Control Concepts for the Alleviation of Windshears and Gusts," NASA CR-166022, July 1982.

${ }^{3}$ Lehman, J. M., Heffley, R. K., and Clement, W. F., "Simulation and Analysis of Wind Shear Hazard," FAA-RD-78-7, Dec. 1977.

"Stengle, R. F., "Optimal Control Laws for Microburst Encounter," Proceedings of the 15th Congress of the ICAS, ICAS-86-5.6.3, London, Sept. 1986.
Belcastro, C. M and Ostroff, A. J., "Tota Energy-Rate Feedback for Automatic Glide-Slope Tracking During Wind-Shear Penetration," NASA TP-2412, May 1985.

${ }^{6}$ König, R.. Krauspe, P., and Schanzer, G.. "Procedures to Improve Flight Safety in Wind Shear Conditions." Proceedings of the 12th Congress of the ICAS, ICAS-80-22.3, Munich, Oct. 1980.

"Miele. A., Wang, T., and Melvin. W. W.. "Oprimization and Gamma/Theta Guidance of Flight Trajectories in a Wind Shear," 15th Congress of the ICAS, ICAS-86-564, London, Sept. 1986.

${ }^{8}$ Miele, A., Wang, T., and Melvin, W. W., "Guidance Strategies for Near-Optimum Take-Off Performance in a Windshear," Journal of Optimization Theory and Applications, Vol. 50, No. 1, 1986. pp. $1-47$.

${ }^{9}$ Miele, A., Wang, T., and Melvin, W. W., "Optimal Flight Trajectories in the Presence of Windshear, Fart 1, Take-Off," AIAA Paper 85-1843, 1985.

${ }^{10}$ Miele, A., Wang, T., and Melvin, W. W., "Optimal Take-Off Trajectories in the Presence of Windshear," Journal of Optimization Theory and Applications, Vol. 49, No. 1, 1986, pp. 1-45.

"Psiaki, M. L., and Stengel, R. F., "Analysis of Aircraft Control Strategies for Microburst Encounter," Journal of Guidance, Control, and Dynamics, Vol. 8, No. 5. 1985, pp. 553-559.

${ }^{12}$ Psiaki, M. L., and Stengel, R. F., "Optimal Flight Paths Through Microburst Wind Profiles," Journat of Aircraft, Vol. 23, No. 8. 1986, pp. 629-635.

'Miele, A., Wang, T., and Melvin. W. W., "Optimization and Guidance of Penetration Landing Trajectories in a Windshear," Proceedings of the American Control Conference, Inst. of Electrical and Electronics Engineers, Piscataway, NJ, 1988, pp. 1428-1439.

${ }^{14}$ Miele, A., Wang, T., and Melvin, W. W., "Optimization and Acceleration Guidance of Flight Trajectories in a Windshear," Journal of Guidance, Control, and Dynamics, Vol. 10, No. 4, 1987, pp. 368-377.

${ }^{15}$ Zhao, Y., and Bryson, A. E., "Optimal Paths Through Downbursts," Proceedings of the AIAA Guidance, Navigation, and Control Conference, AIAA, Washington, DC, 1989, pp. 1139-1149.

${ }^{16}$ Miele, A., Wang, T., Wang, H., and Melvin, W. W. "Overview of Optimal Trajectories for Flight in a Windshear," AIAA Paper 89-0812, 1989

17Psiaki, M. L., "Control of Flight Through Microburst Wind Shear Using Deterministic Trajectory Optimization," Ph.D. Thesis, Princeton Univ., Dept. of Mechanical and Aerospace Engineering. Princeton, NJ, Oct. 1987.

${ }^{18}$ Psiaki, M. L., and Park, K., "Thrust Laws for Microburst Wind Shear Penetration," Proceeding of the AlAA Guidance, Navigation, and Control Conference, AIAA, Washington, DC, 1989. pp. $1127-1138$.

${ }^{19}$ Turkel, B. S., and Frost, W., "Pilot-Aircraft System Response to Wind Shear," NASA CR-3342, Nov. 1980.

${ }^{20}$ Shivers, J. P., Fink, M. P., and Ware, G. M., "Full-Scale Wind Tunnel Investigation of the Static Longitudinal and Lateral Characteristics of a Light, Single-Engine, Low-Wing Airplane," NASA TND-5857, June 1970.

21 "Landing Field Length and Speed," Boeing 727-30 Operations Manual, Boeing, Seattle, WA, Nov. 1977. 